\title{
Relationship of HbA1c with Fasting and Plasma Glucose 2 Hours after Oral Glucose Load in Non Diabetic and Newly Diagnosed Pre Diabetic and Diabetic Patients
}

\author{
HOSSAIN $T^{\mathrm{a}}$, LATIF ZA ${ }^{\mathrm{b}}$, SARKAR AA ${ }^{\mathrm{c}}$
}

\begin{abstract}
:
Objectives: This study was conducted to assess the relationship of Fasting Plasma Glucose and Plasma Glucose 2 hours after 75 gram glucose load with HbA1c in subjects who were not detected before as diabetic, pre diabetic or non diabetic. Methods: Total eight hundred and seventy eight subjects of both sexes coming to do for an Oral Glucose Tolerance Test (OGTT) were included in the study. Fasting and plasma glucose 2 hours after 75 gram glucose load were measured by hexokinase method. HbA1c was measured by high performance liquid chromatography (HPLC) method. Results: According to OGTT among the total study subjects 48.7\%(n=428) had DM, 17.1\% (n=150) had Pre DM and
\end{abstract}

Introduction:

Measuring Glycosylated hemoglobin (HbA1c) is the most important component of the management of diabetes mellitus as it is used to monitor long term glycemic control as well as to guide and adjust therapy. ${ }^{1}$ HbA1c has been well translated to average glucose and mean plasma glucose (MPG). The A1c Derived Average Glucose (ADAG) study defined the mathematical relationship of HbA1c and Average glucose (AG). ${ }^{2}$ HbA1c has been used to assess glycemic control in all major trials, including DCCT and UKPDS, which established the association of HbA1c with the risks of long term complications of hyperglycemia. ${ }^{3,4}$ Recently, HbA1c is also being recommended for diagnosis of diabetes and pre diabetes ${ }^{5}$. For decades, OGTT had been considered as the gold standard test for diagnosis of diabetes and pre diabetes despite of patient's non

a. Dr. Tanjina Hossain, MBBS, MD (EM), Endocrinologist, BIRDEM Hospital, Dhaka.

b. Prof. Zafar Ahmed Latif, MBBS, FCPS (M), Head of the Dept. of Endocrinology, BIRDEM Hospital, Dhaka.

c. Dr. Abdullah Al-mamun Sarkar, MSc (Biochemistry) Research officer, Department of Immunology, BIRDEM Hospital, Dhaka.

Address of Correspondence: Dr. Tanjina Hossain, Endocrinologist, BIRDEM Hospital, Dhaka. Email: tanjina75@gmail.com

Received: 9 February 2012

Accepted: 21 June 2012
$34.2 \%(n=300)$ were non diabetic. Correlation coefficient between Fasting Plasma Glucose and HbA1c was 0.551 (p.000) and correlation coefficient between HbA1c and plasma glucose at 2 hours after oral glucose load was 0.475 (p.000). Conclusion: This study showed moderate degree of relationship between Fasting Plasma Glucose and HbA1c and between HbA1c and plasma glucose 2 hours after 75 gram glucose load in population never diagnosed before as diabetic, pre diabetic or non diabetic.

Keywords: Glycosylated hemoglobin, Fasting plasma glucose, OGTT, Diabetes mellitus, Pre diabetes.

(Birdem Med J 2012; 2(2): 81-83)

adherence to fasting overnight, sample lability and interference by many factors like meal, medication and stress. This study aimed to determine the relationship of HbA1c with Fasting Plasma Glucose and Plasma glucose after 2 hours of 75 gram glucose load in population who were never diagnosed and never treated ,before recommending $\mathrm{HbA} 1 \mathrm{c}$ as an alternative diagnostic method in Bangladeshi population. The study also aimed to evaluate the justification and importance of testing $\mathrm{HbA1C}$ as an initial test during diagnosis of diabetes and pre diabetes.

\section{Methods}

The cross sectional study was carried out in the out patient department of Bangladesh Institute of Research and Rehabilitation in Diabetes, Endocrine and Metabolic Disorders(BIRDEM), Dhaka, Bangladesh during the period from July 2009 to June 2011. The subjects attending BIRDEM blood collection counter 1 and 2 for Oral Glucose Tolerance Test and meeting the inclusion criteria during the study defined period were included in the study. Data was collected on three days a week (every alternate day) from every $5^{\text {th }}$ patient, targeting at least 13 patients/day that resulted the sample size of 936. Among them, 32 samples were clotted, 26 samples were found inadequate, and finally 878 subjects were included in the study. Fasting and Plasma Glucose 2 
hours after 75 gram glucose load were done by hexokinase method. HbA1c was done by High performance Liquid Chromatography (HPLC) method in Bio-Rad system, France. The data was analyzed using Statistical package for Social Science (SPSS) version 17. The strength (degree and extent) and the nature (direction) of relationship between the results had been shown by calculating correlation coefficient(r).

\section{Results}

According to OGTT results and American Diabetes Association, 2010 criteria for diagnosis of diabetes among the total study subjects $48.7 \%(n=428)$ had DM, $17.1 \%(n=150)$ had pre DM and 34.2\% $(n=300)$ were non diabetic (Table-I). Table-II showed F. 2h after Glucose. HbA1c. The age of the subjects in mean \pm SD(SE) 95\% CI was 44.36 $\pm 10.3(0.348) 43.67-45.04$ years. Among the study subjects $50.7 \%(n=445)$ were male and $49.3 \%(n=433)$ were female. The correlation coefficient between Fasting Plasma glucose and HbA1c was 0.551 (p.000). The correlation coefficient between HbA1c and plasma glucose 2 hours after 75 gram glucose load was 0.475 (p.0000).

\section{Table-I}

Disease status among the subjects $(n=878)$, according to OGTT.

\begin{tabular}{lcc} 
Disease status & Frequency (n) & Percentage (\%) \\
\hline DM & 428 & 48.7 \\
Pre DM & 150 & 17.1 \\
Non DM & 300 & 34.2 \\
\hline Total & 878 & 100.0 \\
\hline
\end{tabular}

Table-II

FPG, $2 \mathrm{hPG}$ and HbA1C among the subjects $(n=878)$

\begin{tabular}{lccc} 
Variables & Mean $( \pm$ SD $)$ & $95 \%$ CI & SE \\
\hline FPG & $8.65( \pm 3.27)$ & $8.01-9.29$ & 0.327 \\
2hPG & $14.30( \pm 5.12)$ & $4.27-24.34$ & 0.512 \\
HbA1c & $7.37( \pm 2.42)$ & $7.18-7.50$ & 0.082 \\
\hline
\end{tabular}

\section{Discussion}

The relationship between FPG and HbA1c and between HbA1c and 2 hours PG had been expressed by ' $r$ ' or correlation coefficient. Larger the absolute value of ' $r$ ' stronger the relation and the sign of ' $r$ ' (negative or positive) indicate the nature of relation. In this study ' $r$ ' was 0.551 between FPG and HbA1c, which is larger than that of DCCT ( 0.551 vs 0.82$).{ }^{6}$ The correlation coefficient or ' $r$ ' was 0.475 between HbA1c and PG at 2 hours after oral glucose load. This proved moderate degree of relationship between the variables. The correlation coefficient between FPG and HbA1c and between HbA1c and PG after 2 hours both were statistically significant (p.000).

HbA1c had been used for long time as the most important marker of glycamic control. Major trials, like DCCT showed that only $1 \%$ reduction in HbA1c is associated with marked reduction of all micro vascular and chronic complications of diabetes. Recently, HbA1c has been recommended as a diagnostic marker of diabetes and pre diabetes by American Diabetes Association and this has been accepted by WHO backed group of experts. ${ }^{7}$ HbA1c test has few advantages over OGTT, which is used as the gold standard test for diagnosis of diabetes and pre diabetes since decades. It does not require overnight fasting, can be done at any time of the day and reduces the time of stay at laboratory. It also reduces the problem of taking two blood samples and thus can reduce the work load in laboratories. An initial HbA1c is also recommended to assess the severity of the condition and as a guide of initiating therapy. ${ }^{8}$ The results that were shown in this study may help to prove that measuring HbA1c initially in population who were never diagnosed before as diabetic or pre diabetic may be meaningful and HbA1c can be used as an alternative initial tool to assess the severity of the disease in future in Bangladesh.

\section{Conclusion}

The study revealed that HbA1c has moderate degree of relationship with Fasting Plasma Glucose and Plasma Glucose 2 hours after 75 gram glucose load during an initial test among the population who were never diagnosed and never treated before. It can be concluded that evaluation of $\mathrm{HbA} 1 \mathrm{c}$ as an initial test either alone or along with the OGTT may be helpful for diagnosis, assessment of severity, management and monitoring the prognosis of the disease. 


\section{References:}

1. Sacks DB, Bruns DE, Goldstein DE, Maclaren NK, Mcdonald JM, Parrot M. Guidelines and recommendations for laboratory analysis in the diagnosis and management of diabetes mellitus. Clin Chem 2002;48:436-72.

2. ADAG study Group. Translating the hemoglobin A1c assay into estimated average glucose values. Diabetes Care 2008; 31:1473-78.

3. Diabetes Control and Complications Trial Research Group, 'The effect of intensive treatment of diabetes on development and progression of long term complications in insulin dependant diabetes mellitus', New England Journal of medicine, 1993; 329: 977-86.

4. UK Prospective Diabetes Study Group, Intensive blood glucose with sulphonylureas or insulin compared with conventional treatment and risk of complications in patients with type 2 diabetes', Lancet, 1998; 352: 83753.

5. American Diabetes Association, 'Standards of medical care in diabetes-2010’, Diabetes Care, 2010; 33(1) : S11-S61.

6. Rohlfing CL, Wiedmeyer HM, Little R, England JD, Tennill A, goldstein DE. Defining the relationship between plasma glucose and HbA1c in the Diabetes Control and Complications Trial. Diabetes Care 2002; 25:275-8.

7. WHO expert consultation Report, 2011, Retrieved from, ht tp://www.who.int/chp/media/news/release/ 2011_1_1diabetes/en/

8. American Association for Clinical Endocrinologists (AACE) Diabetes Care Plan Guidelines, Endocr Prac. 2011;17 (suppl 2). 